\title{
Matched/Mismatched Interaction of a Cyclic Hexapeptide with Ion Pairs Containing Chiral
}

\section{Cations and Chiral Anions}

\author{
Guido Heinrichs, ${ }^{\dagger}$ Stefan Kubik*, ${ }^{\dagger}$, Jérôme Lacour, ${ }^{\S}$ Laurent Vial ${ }^{\S}$ \\ ${ }^{\dagger}$ Fachbereich Chemie - Organische Chemie, Technische Universität Kaiserlautern, Erwin- \\ Schrödinger-Straße, D-67663 Kaiserslautern, Germany., Fax: +49-631-205-3921, Email: \\ kubik@chemie.uni-kl.de \\ ${ }^{\S}$ Département de Chimie Organique, Université de Genève, Quai Ernest Ansermet 30, \\ CH-1211 Genève 4, Switzerland
}

\section{CONTENT}

Structures of the compounds investigated................................................................... 2

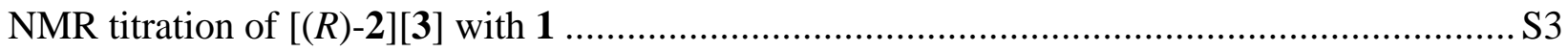

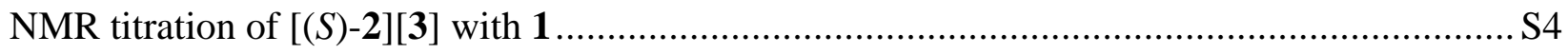

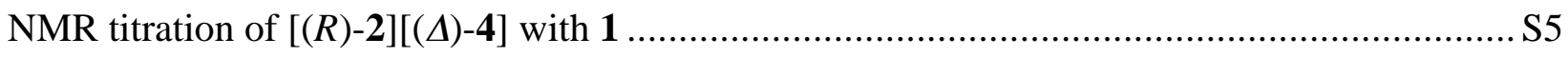

NMR titration of 1 with $[(R)-2][(\Delta)-4]$ following the shift of the NH signal of the host ............ S6

NMR titration of 1 with $[(R)-2][(\Delta)-4]$ following the shift of the $\mathrm{H}(\alpha)$ signal of the host..........S7

NMR titration of $\mathbf{1}$ with $[(R)-2][(\Delta)-4]$ following the shift of an aromatic signal of the host ...... S8

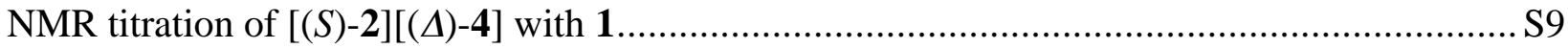

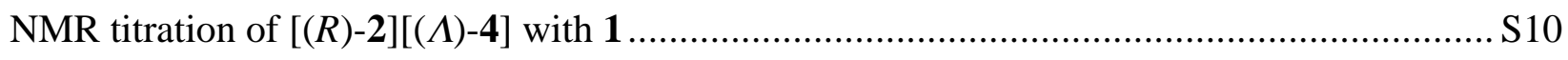

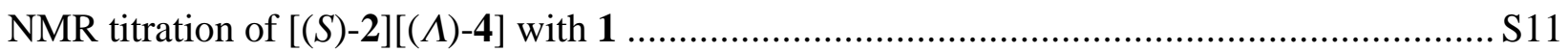

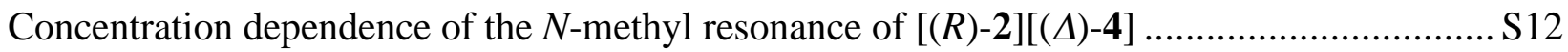


$\underline{\text { Structures of the compounds investigated: }}$
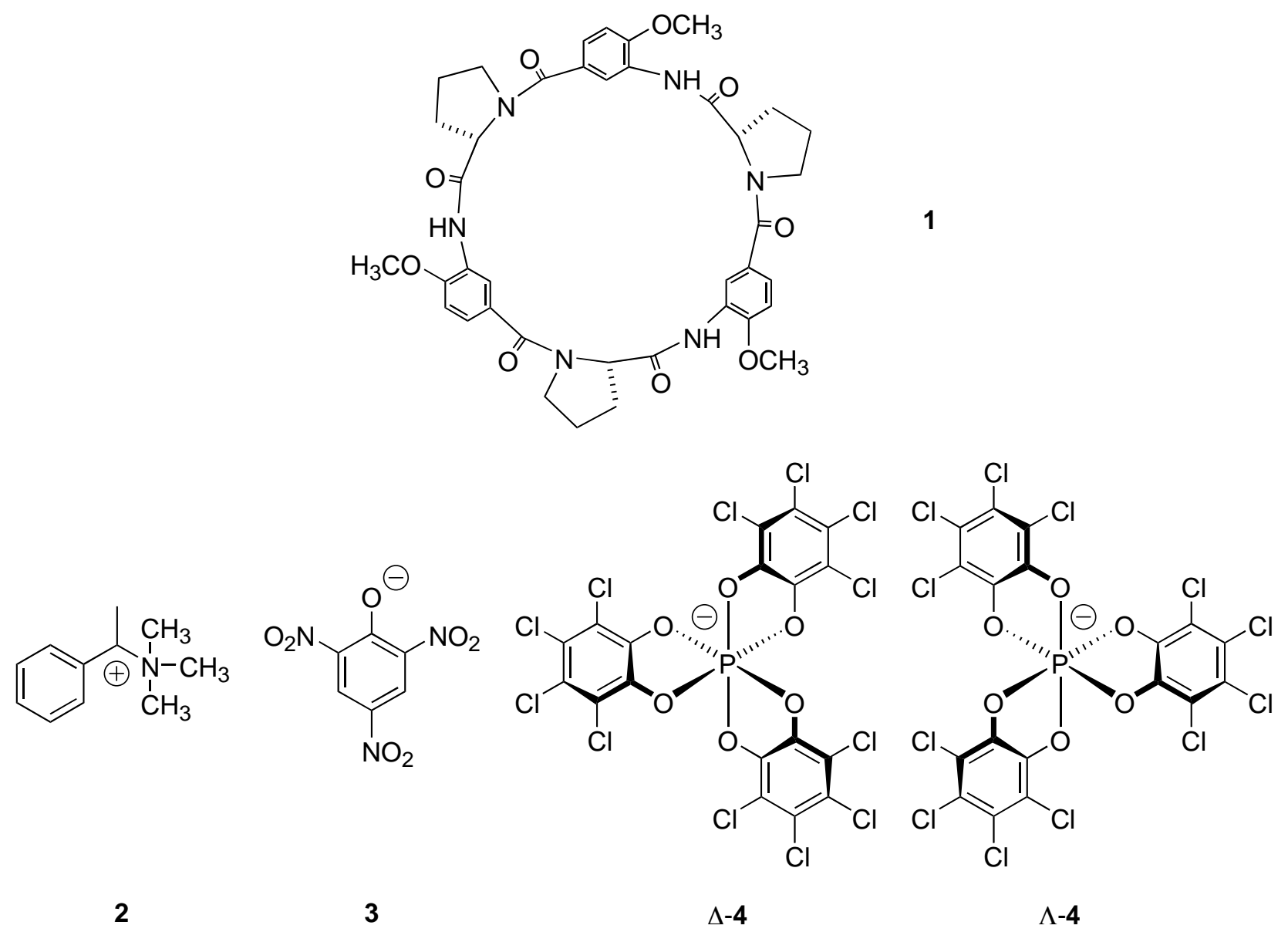
NMR titration of $[(R)-2][3]$ with 1 following the shift of the $N$-methyl signal of the cation:

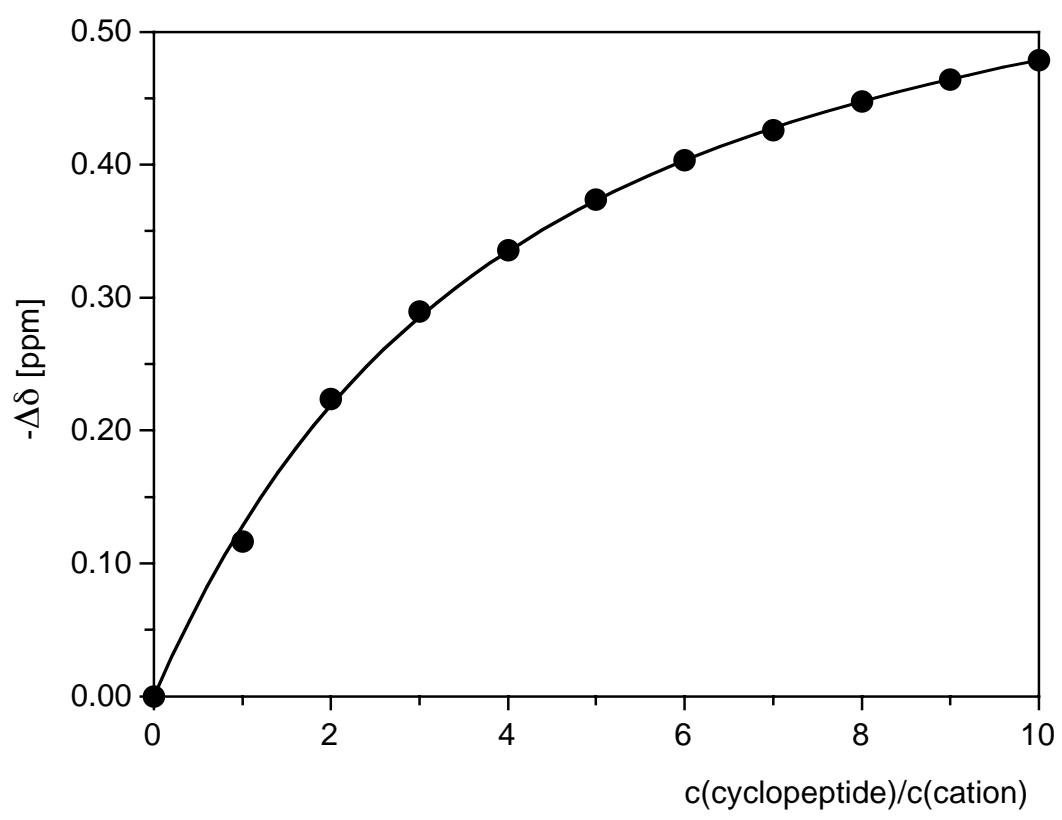

Results of the non-linear regression: $K_{\mathrm{a}}=1550 \mathrm{M}^{-1}, \Delta \delta_{\max }=-0.646$

Concentration dependence of $K_{\mathrm{a}}$ :

\begin{tabular}{ccccc}
\hline$[1]_{0} / \mathrm{mM}$ & {$[\mathrm{salt}]_{0} / \mathrm{mM}$} & $-\Delta \delta$ & $\Delta \delta / \Delta \delta_{\max }$ & $K_{\mathrm{a}} / \mathrm{M}^{-1}$ \\
\hline 0.20 & 0.20 & 0.117 & 0.180 & 1340 \\
0.40 & 0.20 & 0.224 & 0.347 & 1600 \\
0.60 & 0.20 & 0.289 & 0.448 & 1590 \\
0.80 & 0.20 & 0.336 & 0.519 & 1550 \\
1.00 & 0.20 & 0.374 & 0.578 & 1550 \\
1.20 & 0.20 & 0.404 & 0.625 & 1550 \\
1.40 & 0.20 & 0.426 & 0.659 & 1530 \\
1.60 & 0.20 & 0.447 & 0.693 & 1540 \\
1.80 & 0.20 & 0.464 & 0.719 & 1540 \\
2.00 & 0.20 & 0.479 & 0.741 & 1550 \\
\hline & & & Average: & 1560 \\
& & & Deviation: & 20 \\
\hline
\end{tabular}


NMR titration of $[(S)-2][3]$ with 1 following the shift of the $N$-methyl signal of the cation:

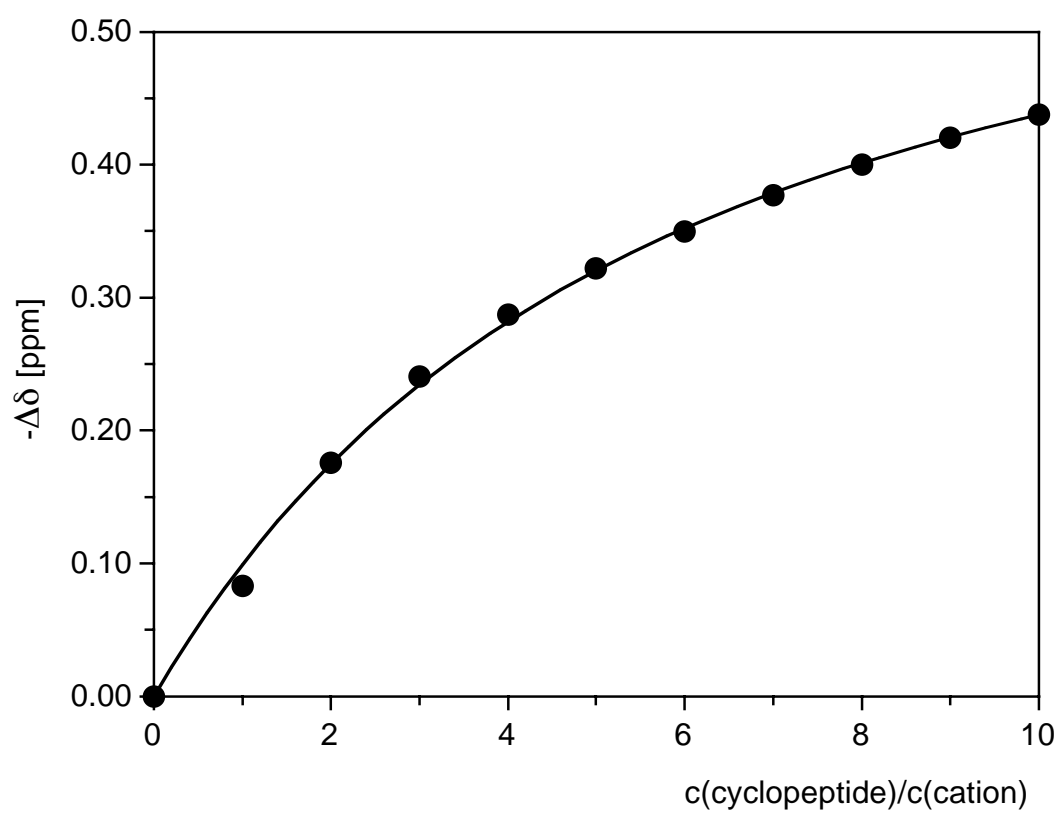

Results of the non-linear regression: $K_{\mathrm{a}}=1030 \mathrm{M}^{-1}, \Delta \delta_{\max }=-0.666$

Concentration dependence of $K_{\mathrm{a}}$ :

\begin{tabular}{ccccc}
\hline$[1]_{0} / \mathrm{mM}$ & {$[\mathrm{salt}]_{0} / \mathrm{mM}$} & $-\Delta \delta$ & $\Delta \delta / \Delta \delta_{\max }$ & $K_{\mathrm{a}} / \mathrm{M}^{-1}$ \\
\hline 0.20 & 0.20 & 0.083 & 0.125 & 810 \\
0.40 & 0.20 & 0.176 & 0.264 & 1030 \\
0.60 & 0.20 & 0.241 & 0.361 & 1070 \\
0.80 & 0.20 & 0.287 & 0.431 & 1060 \\
1.00 & 0.20 & 0.322 & 0.484 & 1040 \\
1.20 & 0.20 & 0.350 & 0.526 & 1010 \\
1.40 & 0.20 & 0.377 & 0.566 & 1020 \\
1.60 & 0.20 & 0.400 & 0.601 & 1020 \\
1.80 & 0.20 & 0.420 & 0.631 & 1020 \\
2.00 & 0.20 & 0.438 & 0.658 & 1030 \\
\hline & & & Average: & 1030 \\
& & & Deviation: & 20 \\
\hline
\end{tabular}


NMR titration of $[(R)-2][(\Delta)-4]$ with 1 following the shift of the $N$-methyl signal of the cation:

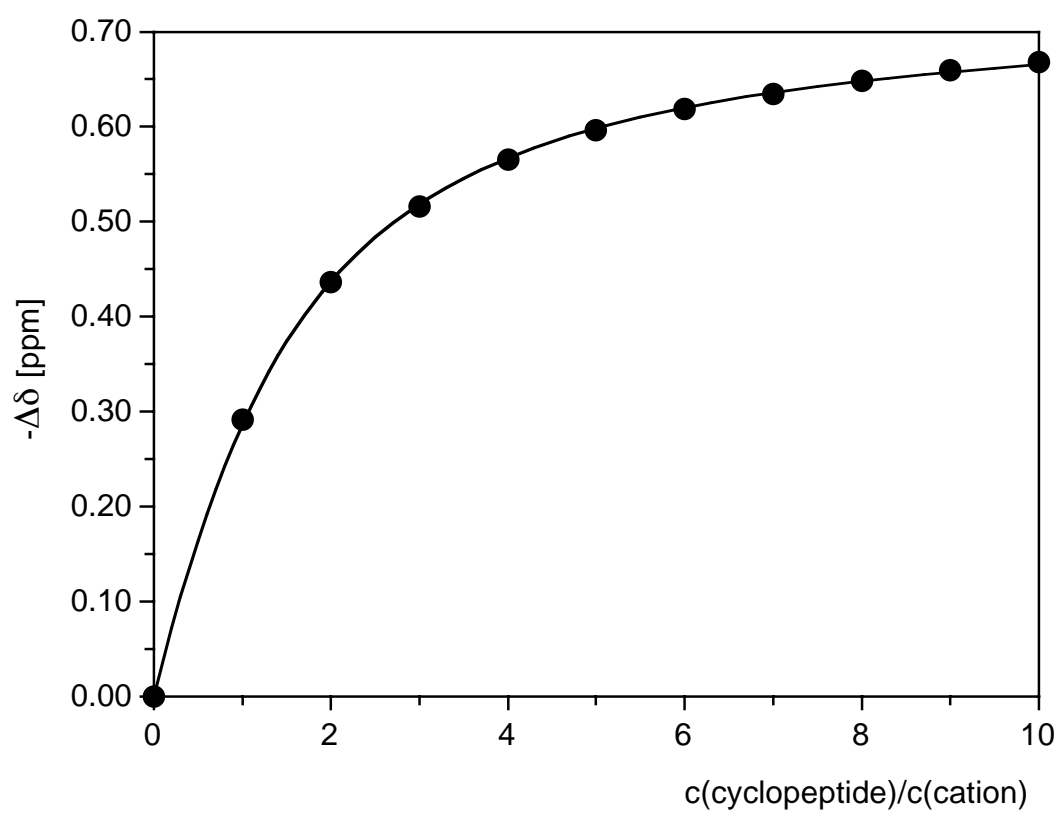

Results of the non-linear regression: $K_{\mathrm{a}}=5210 \mathrm{M}^{-1}, \Delta \delta_{\max }=-0.736$

Concentration dependence of $K_{\mathrm{a}}$ :

\begin{tabular}{ccccc}
\hline$[1]_{0} / \mathrm{mM}$ & {$[\mathrm{salt}]_{0} / \mathrm{mM}$} & $-\Delta \delta$ & $\Delta \delta / \Delta \delta_{\max }$ & $K_{\mathrm{a}} / \mathrm{M}^{-1}$ \\
\hline 0.20 & 0.20 & 0.291 & 0.396 & 5430 \\
0.40 & 0.20 & 0.437 & 0.594 & 5190 \\
0.60 & 0.20 & 0.516 & 0.702 & 5120 \\
0.80 & 0.20 & 0.565 & 0.769 & 5140 \\
1.00 & 0.20 & 0.596 & 0.811 & 5110 \\
1.20 & 0.20 & 0.619 & 0.841 & 5140 \\
1.40 & 0.20 & 0.635 & 0.863 & 5140 \\
1.60 & 0.20 & 0.648 & 0.882 & 5230 \\
1.80 & 0.20 & 0.660 & 0.897 & 5360 \\
2.00 & 0.20 & 0.668 & 0.908 & 5450 \\
\hline & & & Average: & 5210 \\
& & & Deviation: & 110 \\
\hline
\end{tabular}


NMR titration of 1 with $[(R)-2][(\Delta)-4]$ following the shift of the NH signal of the host:

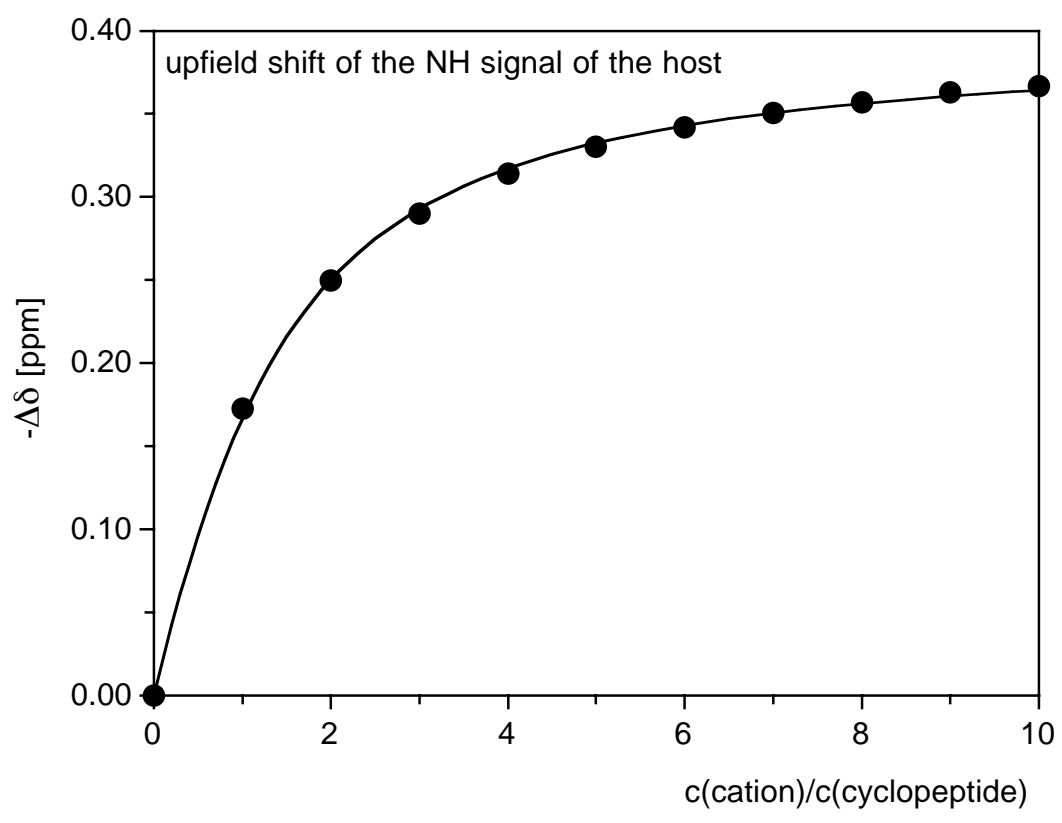

Results of the non-linear regression: $K_{\mathrm{a}}=5010 \mathrm{M}^{-1}, \Delta \delta_{\max }=-0.397$

Concentration dependence of $K_{\mathrm{a}}$ :

\begin{tabular}{ccccc}
\hline$[\mathbf{1}]_{0} / \mathrm{mM}$ & {$[\mathrm{salt}]_{0} / \mathrm{mM}$} & $-\Delta \delta$ & $\Delta \delta / \Delta \delta_{\max }$ & $K_{\mathrm{a}} / \mathrm{M}^{-1}$ \\
\hline 0.25 & 0.25 & 0.173 & 0.436 & 5480 \\
0.25 & 0.50 & 0.250 & 0.630 & 4970 \\
0.25 & 0.75 & 0.290 & 0.732 & 4810 \\
0.25 & 1.00 & 0.314 & 0.792 & 4750 \\
0.25 & 1.25 & 0.330 & 0.834 & 4810 \\
0.25 & 1.50 & 0.342 & 0.862 & 4870 \\
0.25 & 1.75 & 0.351 & 0.884 & 5010 \\
0.25 & 2.00 & 0.357 & 0.900 & 5090 \\
0.25 & 2.25 & 0.363 & 0.916 & 5410 \\
0.25 & 2.50 & 0.367 & 0.926 & 5480 \\
\hline & & & Average: & 5020 \\
& & & Deviation: & 250 \\
\hline
\end{tabular}


NMR titration of 1 with $[(R)-2][(\Delta)-4]$ following the shift of the $H(\alpha)$ signal of the host:

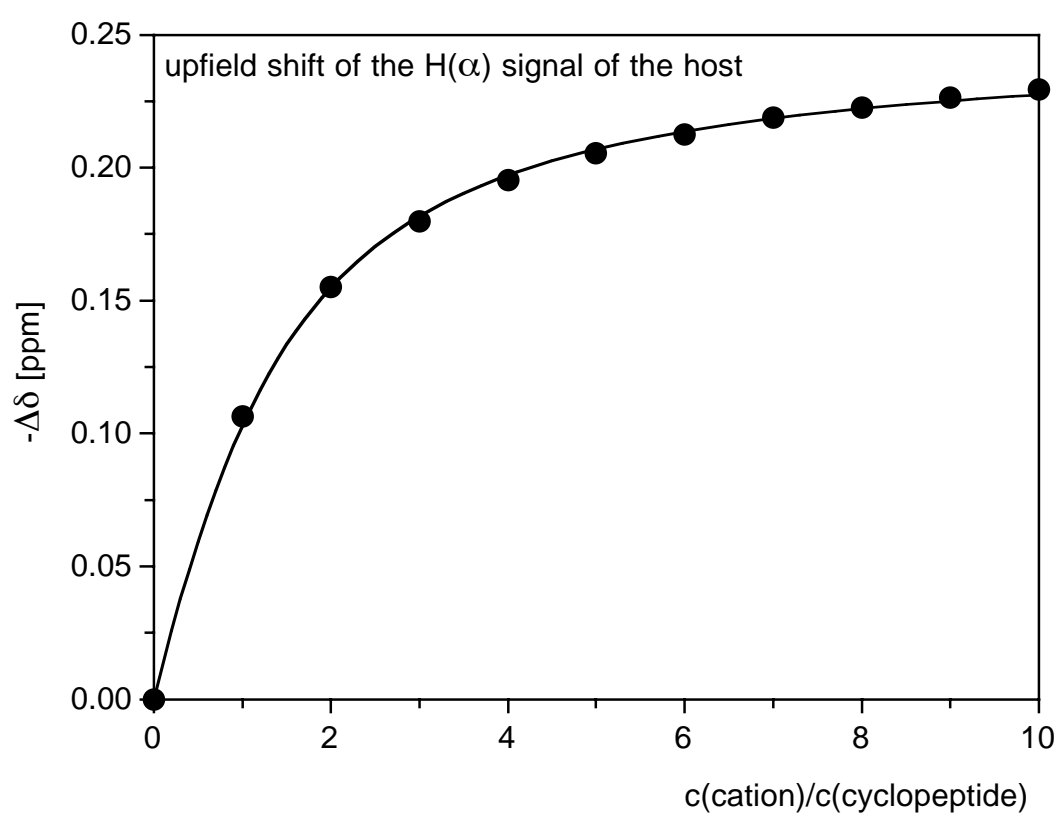

Results of the non-linear regression: $K_{\mathrm{a}}=4830 \mathrm{M}^{-1}, \Delta \delta_{\max }=-0.248$

Concentration dependence of $K_{\mathrm{a}}$ :

\begin{tabular}{ccccc}
\hline$[\mathbf{1}]_{0} / \mathrm{mM}$ & {$[\mathrm{salt}]_{0} / \mathrm{mM}$} & $-\Delta \delta$ & $\Delta \delta / \Delta \delta_{\max }$ & $K_{\mathrm{a}} / \mathrm{M}^{-1}$ \\
\hline 0.25 & 0.25 & 0.107 & 0.429 & 5270 \\
0.25 & 0.50 & 0.155 & 0.625 & 4850 \\
0.25 & 0.75 & 0.180 & 0.724 & 4620 \\
0.25 & 1.00 & 0.195 & 0.788 & 4620 \\
0.25 & 1.25 & 0.206 & 0.828 & 4630 \\
0.25 & 1.50 & 0.213 & 0.857 & 4640 \\
0.25 & 1.75 & 0.219 & 0.882 & 4880 \\
0.25 & 2.00 & 0.223 & 0.897 & 4890 \\
0.25 & 2.25 & 0.226 & 0.912 & 5130 \\
0.25 & 2.50 & 0.230 & 0.925 & 5440 \\
\hline & & & Average: & 4860 \\
& & & Deviation: & 270 \\
\hline
\end{tabular}


NMR titration of 1 with $[(R)-2][(\Delta)-4]$ following the shift of an aromatic signal of the host:

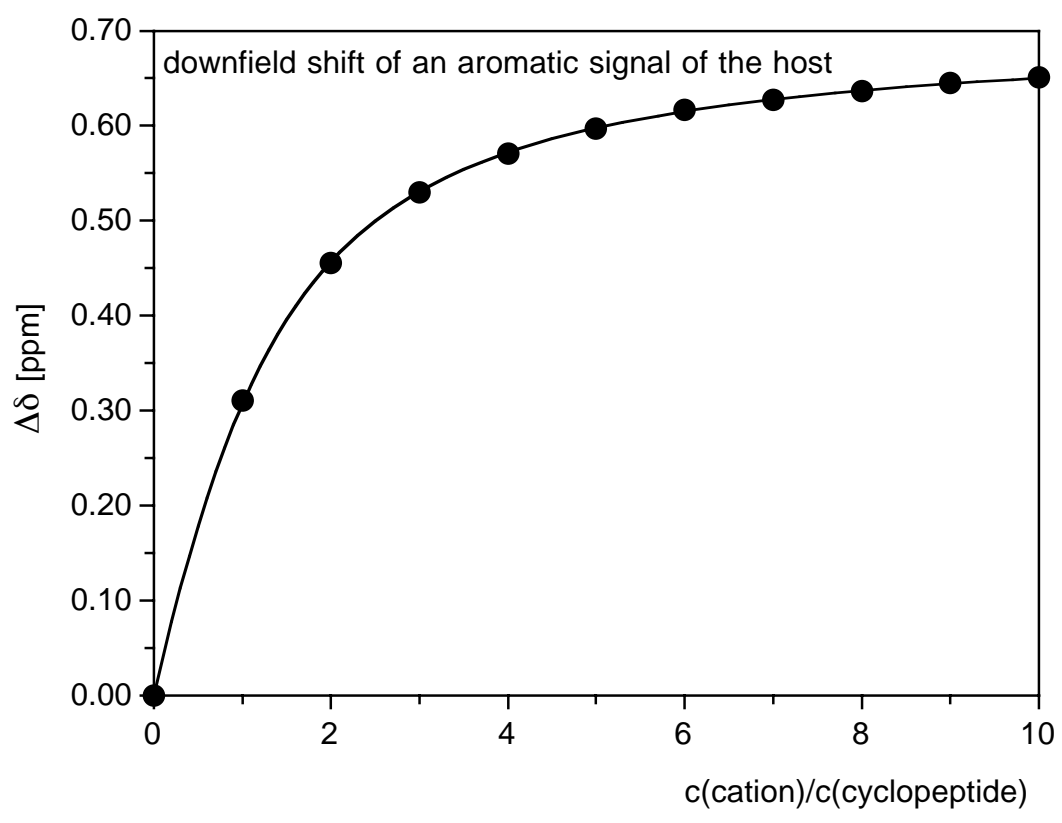

Results of the non-linear regression: $K_{\mathrm{a}}=5520 \mathrm{M}^{-1}, \Delta \delta_{\max }=0.702$

Concentration dependence of $K_{\mathrm{a}}$ :

\begin{tabular}{ccccc}
\hline$[\mathbf{1}]_{0} / \mathrm{mM}$ & {$[\mathrm{salt}]_{0} / \mathrm{mM}$} & $\Delta \delta$ & $\Delta \delta / \Delta \delta_{\max }$ & $K_{\mathrm{a}} / \mathrm{M}^{-1}$ \\
\hline 0.25 & 0.25 & 0.310 & 0.442 & 5670 \\
0.25 & 0.50 & 0.455 & 0.648 & 5450 \\
0.25 & 0.75 & 0.530 & 0.754 & 5460 \\
0.25 & 1.00 & 0.571 & 0.813 & 5440 \\
0.25 & 1.25 & 0.597 & 0.850 & 5480 \\
0.25 & 1.50 & 0.617 & 0.879 & 5660 \\
0.25 & 1.75 & 0.627 & 0.893 & 5490 \\
0.25 & 2.00 & 0.636 & 0.906 & 5450 \\
0.25 & 2.25 & 0.645 & 0.919 & 5580 \\
0.25 & 2.50 & 0.651 & 0.928 & 5640 \\
\hline & & & Average: & 5520 \\
& & & Deviation: & 80 \\
\hline
\end{tabular}


NMR titration of $[(S)-2][(\Delta)-4]$ with 1 following the shift of the $N$-methyl signal of the cation:

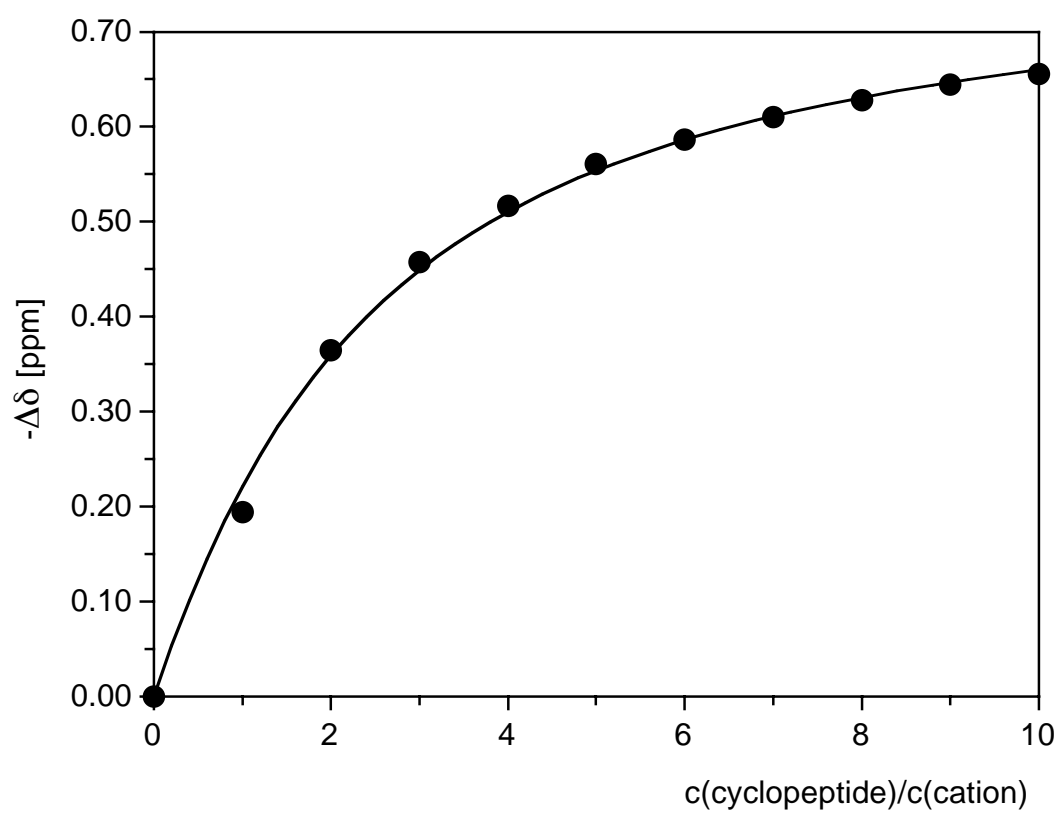

Results of the non-linear regression: $K_{\mathrm{a}}=2660 \mathrm{M}^{-1}, \Delta \delta_{\max }=-0.795$

Concentration dependence of $K_{\mathrm{a}}$ :

\begin{tabular}{ccccc}
\hline$[1]_{0} / \mathrm{mM}$ & {$[\mathrm{salt}]_{0} / \mathrm{mM}$} & $-\Delta \delta$ & $\Delta \delta / \Delta \delta_{\max }$ & $K_{\mathrm{a}} / \mathrm{M}^{-1}$ \\
\hline 0.20 & 0.20 & 0.194 & 0.244 & 2140 \\
0.40 & 0.20 & 0.364 & 0.458 & 2740 \\
0.60 & 0.20 & 0.458 & 0.576 & 2800 \\
0.80 & 0.20 & 0.517 & 0.650 & 2770 \\
1.00 & 0.20 & 0.561 & 0.706 & 2790 \\
1.20 & 0.20 & 0.586 & 0.737 & 2670 \\
1.40 & 0.20 & 0.610 & 0.767 & 2650 \\
1.60 & 0.20 & 0.628 & 0.790 & 2600 \\
1.80 & 0.20 & 0.644 & 0.810 & 2610 \\
2.00 & 0.20 & 0.656 & 0.824 & 2560 \\
\hline & & & Average: & 2690 \\
& & & Deviation: & 90 \\
\hline
\end{tabular}


NMR titration of $[(R)-2][(\Lambda)-4]$ with 1 following the shift of the $N$-methyl signal of the cation:

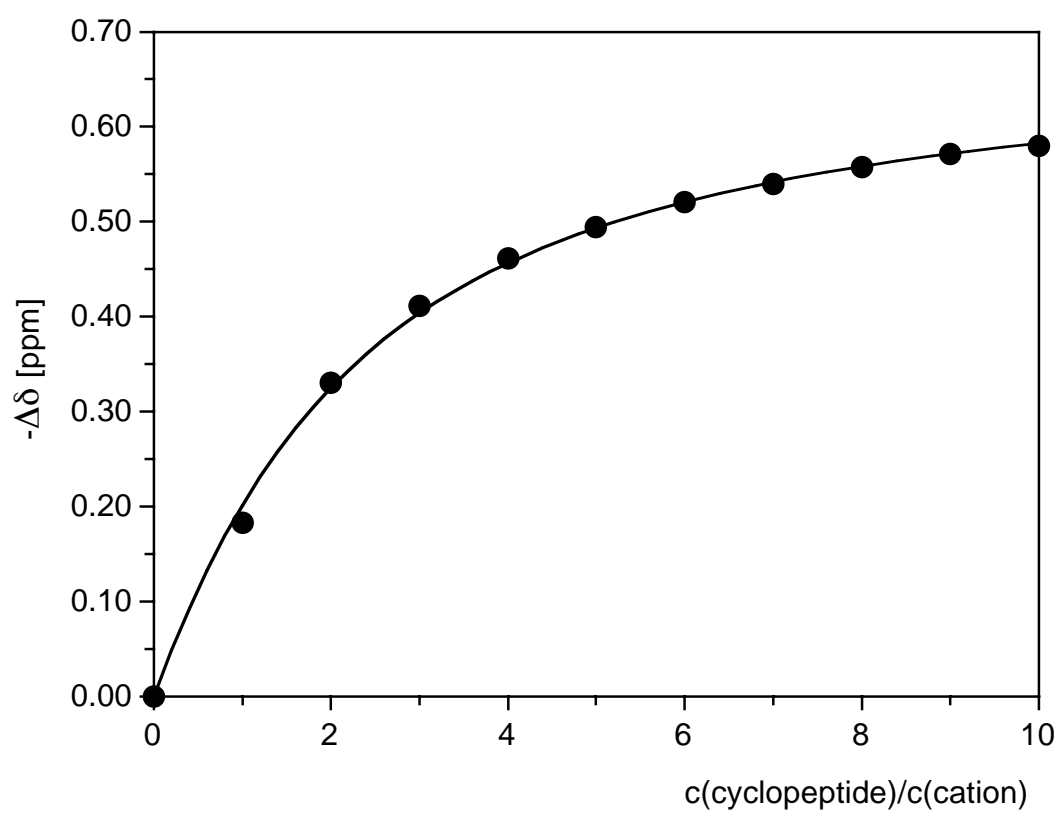

Results of the non-linear regression: $K_{\mathrm{a}}=2890 \mathrm{M}^{-1}, \Delta \delta_{\max }=-0.692$

Concentration dependence of $K_{\mathrm{a}}$ :

\begin{tabular}{ccccc}
\hline$[1]_{0} / \mathrm{mM}$ & {$[\mathrm{salt}]_{0} / \mathrm{mM}$} & $-\Delta \delta$ & $\Delta \delta / \Delta \delta_{\max }$ & $K_{\mathrm{a}} / \mathrm{M}^{-1}$ \\
\hline 0.20 & 0.20 & 0.183 & 0.264 & 2440 \\
0.40 & 0.20 & 0.330 & 0.477 & 3000 \\
0.60 & 0.20 & 0.411 & 0.594 & 3040 \\
0.80 & 0.20 & 0.462 & 0.667 & 3000 \\
1.00 & 0.20 & 0.494 & 0.714 & 2910 \\
1.20 & 0.20 & 0.521 & 0.752 & 2890 \\
1.40 & 0.20 & 0.540 & 0.779 & 2840 \\
1.60 & 0.20 & 0.557 & 0.805 & 2870 \\
1.80 & 0.20 & 0.571 & 0.825 & 2880 \\
2.00 & 0.20 & 0.580 & 0.838 & 2820 \\
\hline & & & Average: & 2920 \\
& & & Deviation: & 70 \\
\hline
\end{tabular}


NMR titration of $[(S)-2][(\Lambda)-4]$ with 1 following the shift of the $N$-methyl signal of the cation:

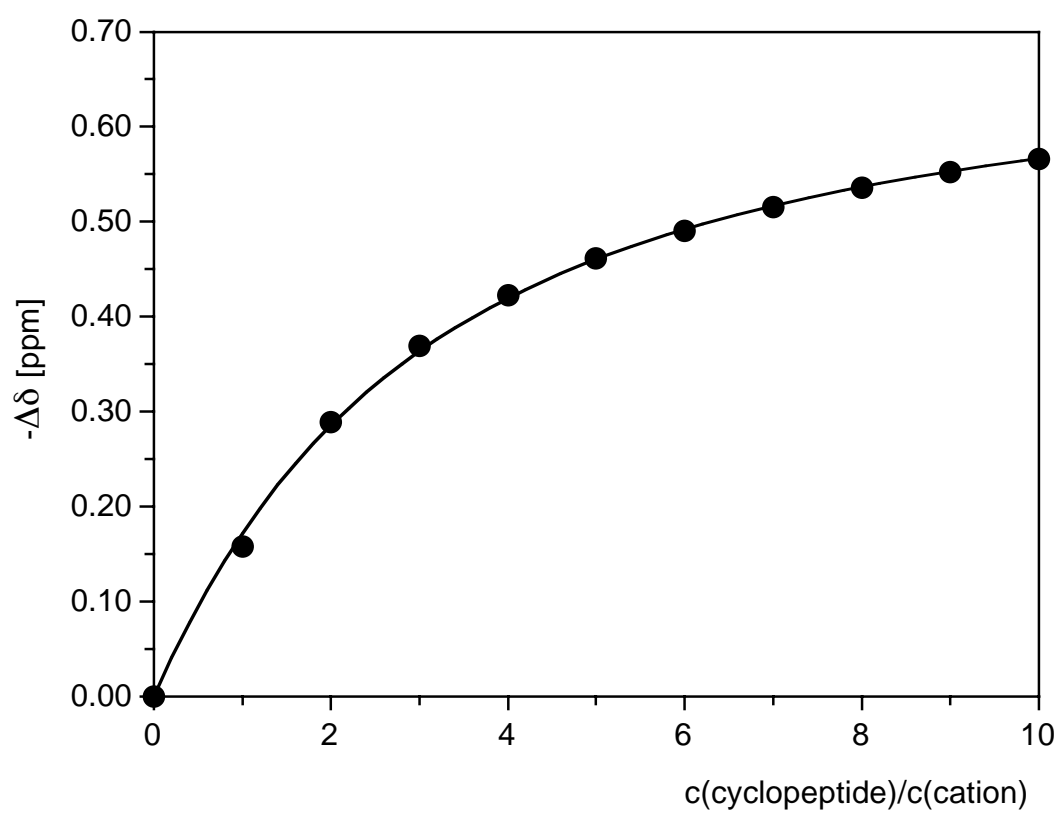

Results of the non-linear regression: $K_{\mathrm{a}}=2080 \mathrm{M}^{-1}, \Delta \delta_{\max }=-0.715$

Concentration dependence of $K_{\mathrm{a}}$ :

\begin{tabular}{ccccc}
\hline$[1]_{0} / \mathrm{mM}$ & {$[\mathrm{salt}]_{0} / \mathrm{mM}$} & $-\Delta \delta$ & $\Delta \delta / \Delta \delta_{\max }$ & $K_{\mathrm{a}} / \mathrm{M}^{-1}$ \\
\hline 0.20 & 0.20 & 0.158 & 0.221 & 1820 \\
0.40 & 0.20 & 0.289 & 0.404 & 2120 \\
0.60 & 0.20 & 0.369 & 0.517 & 2150 \\
0.80 & 0.20 & 0.422 & 0.591 & 2120 \\
1.00 & 0.20 & 0.461 & 0.646 & 2090 \\
1.20 & 0.20 & 0.490 & 0.686 & 2060 \\
1.40 & 0.20 & 0.516 & 0.721 & 2060 \\
1.60 & 0.20 & 0.536 & 0.750 & 2070 \\
1.80 & 0.20 & 0.552 & 0.773 & 2070 \\
2.00 & 0.20 & 0.566 & 0.792 & 2070 \\
\hline & & & Average: & 2090 \\
& & & Deviation: & 30 \\
\hline
\end{tabular}


Concentration dependence of the $N$-methyl resonance of $[(R)-2][(\Delta)-4]$ :

\begin{tabular}{cc}
\hline$[$ salt $] / \mathrm{mM}$ & $\delta$ \\
\hline 2.00 & 3.262 \\
1.00 & 3.265 \\
0.50 & 3.268 \\
0.20 & 3.269 \\
0.10 & 3.268 \\
0.05 & 3.268 \\
0.02 & 3.267 \\
0.02 & 3.268 \\
\hline
\end{tabular}

All measurements were carried out in $0.1 \% d_{6}-\mathrm{DMSO} / \mathrm{CDCl}_{3}$. 